\title{
Multi-layer Model Predictive Control of Regional Water Networks: Application to the Catalunya Case Study*
}

\author{
Cong Cong Sun ${ }^{1}$, Vicenç Puig ${ }^{2}$ and Gabriela Cembrano ${ }^{3}$
}

\begin{abstract}
A multi-layer model predictive control (MPC) scheme with temporal multi-level coordination for regional water networks is proposed. First, a multi-layer control structure resulting from a functional decomposition of water network is briefly presented. Inside each layer, a MPC based controller is used. Between related layers, a temporal multi-level coordination mechanism is used to generate control strategies which consider objectives and time scales of both layers. The proposed approach will be applied to the Catalunya Regional Water Network. The paper presents the results based on an aggregate model of this network.
\end{abstract}

\section{INTRODUCTION}

From a functional perspective, a regional water network can be structurally organized into three layers [1]:

- Supply layer, which is the upper layer, composed by water sources, large reservoirs and also natural aquifers.

- Transportation layer, the middle layer, links water treatment with reservoirs distributed all over the city.

- Distribution layer, the lower layer used for meeting consumer demands.

Each of the layers of a regional water network must be operated at different time scale because of the different dynamics they present according to their specified objectives. In general, these layers are often separately operated and the ecological effect, also sustainable usages of water has seldom been included [1]-[3]. The coordinated operation of different layers in a regional network is one of the main motivations for the research in this paper.

MPC has been proven to be one of the most effective control strategies for the global optimal operational control of large-scale water networks [1], [4], [5].

The main contribution of this paper is proposing a temporal multi-layer hierarchical MPC scheme for regional water networks. The proposed strategy will coordinate the MPC controllers for the supply and transportation layers by means of a temporal hierarchical sequence of optimizations and constraints going from the upper to the lower layer.

The paper is organized as follows: Section II introduces the control oriented modelling methodology proposed for regional water networks. In Section III, MPC, multi-layer MPC and temporal multi-level coordination techniques are

\footnotetext{
*This work has been funded by the Spanish Ministry of Science and Technology through the project CYCYT WATMAN DPI2009-13744 and by EFFINET grant FP7-ICT-2012-318556 of the European Commission

${ }^{1}$ Cong Cong Sun, Vicenç Puig and Gabriela Cembrano are with Advanced Control Systems Group at the Institut de Robòtica i Informàtica Industrial (CSIC-UPC), Llorens i Artigas, 4-6, 08028 Barcelona, Spain. e-mail:vicenc.puig@upc.edu
}

outlined. In Section IV, the formulation of MPC and temporal multi-level coordination is presented. In Section V, the considered case study based on the Catalunya Regional Water Network is described. In Section VI, the application results of the proposed modelling and controlling approach to Catalunya Regional Water Network are outlined. Finally, in Section VII, the main conclusions are presented.

\section{CONTROL ORIENTED MODELLING METHODOLOGY}

A water system generally contains tanks, a network of links(pipes and open-flow channels) and a number of demands. Valves and/or pumping stations are elements that allow to manipulate the water flow according to a specific policy and to supply water requested by the network users.

\section{A. Tanks and Reservoirs}

The mass balance expression relating the stored volume $v$, the manipulated inflows $q_{\text {in }}^{j}$ and outflows $q_{\text {out }}^{h}$ (including the demand flows as outflows) for the $i$-th tank can be written as:

$$
v_{i}(k+1)=v_{i}(k)+\Delta t\left(\sum_{j} q_{\mathrm{in}}^{j}(k)-\sum_{h} q_{\mathrm{out}}^{h}(k)\right)
$$

where $\Delta t$ is the sampling time and $k$ denotes the discretetime instant. The physical constraint related to the range of admissible water in the $i$-th tank is expressed as

$$
\underline{v}_{i} \leq v_{i}(k) \leq \bar{v}_{i}, \quad \text { for all } k
$$

where $\underline{v}_{i}$ and $\bar{v}_{i}$ denote minimum and maximum storage.

\section{B. Actuators}

The manipulated flows through actuators represent manipulated variables, denoted as $q_{u}$. Both have lower and upper limits, which are taken as system constraints. As in (2), they are expressed as

$$
\underline{q u}_{i} \leq q_{u_{i}}(k) \leq \overline{q_{u}}, \quad \text { for all } k
$$

where $\underline{q u}_{i}$ and $\overline{q_{u}}$ denote minimum and maximum flow.

\section{Nodes}

These elements correspond to the network points where water flows are merged or split. Thus, the nodes represent mass balance relations, being modelled as equality constraints related to inflows and outflows. Expression of the mass conservation in these elements can be written as

$$
\sum_{j} q_{\text {in }}^{j}(k)=\sum_{h} q_{\text {out }}^{h}(k)
$$




\section{River Reaches}

A single canal reach can be approximated by using the IDZ model in literature [6] and [7] as follows:

$$
Y_{d n s}(s)=G_{1}(s) Q_{u p s}(s)+G_{2}(s) Q_{d n s}(s)
$$

where $Y_{d n s}(s)$ is water level at the control point, and $Q_{u p s}(s), Q_{d n s}(s)$ are the upstream and downstream flows, respectively. Moreover, $G_{1}(s)=e^{-\tau_{d} s} /\left(A_{d} s\right)$ and $G_{2}(s)=$ $-1 /\left(A_{d} s\right)$ with $\tau_{d}$ being the downstream transport delay and $A_{d}$ the downstream backwater area.

Taking into account the linearized relation between $Q_{d n s}$ and $Y_{d n s}$, the following relation can be established:

$$
Q_{d n s}(s)=\beta Y_{d n s}(s)
$$

where $\beta$ is a constant. Combining (5) and (6), the following first order plus time delay (FOPTD) model is obtained

$$
G(s)=\frac{Q_{d n s}(s)}{Q_{u p s}(s)}=\frac{K e^{-\tau_{d} s}}{T s+1}
$$

with $K=1$ and $T=A_{d} / \beta$. This model can be represented in discrete-time, using a sampling time $\Delta t$, as follows:

$$
G_{d}(z)=\frac{Q_{d n s}(z)}{Q_{u p s}(z)}=\frac{b_{0} z^{-d}}{z-a_{1}}
$$

where $d=\operatorname{round}\left(\tau_{d}\right) / T_{s}, b_{0}=1-a_{1}$ and $a_{1}=e^{-\frac{T_{s}}{T}}$. Alternatively, it can be written as a difference equation as

$$
q_{d n s}(k+1)=a_{1} q_{d n s}(k)+b_{0} q_{\text {ups }}(k-d)
$$

\section{E. Demand and Irrigation Sectors}

Demand and irrigation sectors (simplified as water demand) represent water demands made by network users. That are considered as measured disturbances of the system at a given time instant. The demand can be anticipated by a forecasting algorithm [8] which is integrated and run in parallel with MPC closed-loop architecture.

\section{TEMPORAL MULTI-LAYER MPC SCHEME}

\section{A. Model Predictive Control}

The standard MPC problem based on the linear discretetime prediction model is considered as described in [9]:

$$
\begin{aligned}
x(k+1) & =A x(k)+B u(k) \\
y(k) & =C x(k)
\end{aligned}
$$

where $x(k) \in \mathbb{R}^{n_{x}}$ is state vector and $u(k) \in \mathbb{R}^{n_{u}}$ is vector of command variables, while $y(k) \in \mathbb{R}^{n_{y}}$ is vector of the measured output. Following the formalism provided by [9], the cost function is assumed to be quadratic and the constraints are in the form of linear inequalities, which constitute the following basic optimization problem (BOP):

$$
\begin{array}{ll} 
& \min _{\text {s.t. }} J(k) \\
& x(i+1 \mid k)=A x(i \mid k)+B u(i \mid k), \quad i=1, \cdots, H_{p} \\
& x(0 \mid k)=x_{k} \\
& x_{\min } \leq x(i \mid k) \leq x_{\max }, \quad i=1, \cdots, H_{p} \\
& u_{\min } \leq u(i \mid k) \leq u_{\max }, \quad i=0, \cdots, H_{p}-1
\end{array}
$$

As described above $J$ is a performance index, representing operational goals of the system, $H_{p}$ is the prediction horizon, $x(0)$ is the initial condition of the state vector, $x_{\min }$ and $x_{\max }$ are minimal and maximal capacities of tanks, while $u_{\min }$ and $u_{\max }$ are known vectors defining the saturation constraints on inputs variables. MPC Problem (11) can be recast as a Quadratic Programming (QP) problem, whose solution:

$$
\mathcal{U}^{*}(k) \triangleq\left[u^{*}(0 \mid k) \cdots u^{*}\left(H_{p}-1 \mid k\right)\right]^{T} \in \mathbb{R}^{H_{p} m \times 1}
$$

is a sequence of optimal control inputs that generates an admissible state sequence. At each sampling time $k, M P C$ Problem (11) is solved for the given measured/estimated current state $x(k)$. Only the first optimal move $u^{*}(0 \mid k)$ of the optimal sequence $\mathcal{U}^{*}(k)$ is applied to the process:

$$
u_{M P C}(k)=u^{*}(0 \mid k)
$$

the remaining optimal moves are discarded and the optimization is repeated at time $k+1$.

\section{B. Multi-layer Model Predictive Control}

This paper proposes a temporal multi-layer MPC scheme for the control of regional water networks [10].

The general principle of temporal multi-layer MPC is that decision of a higher layer have a wider temporal extent than the one of a lower layer. At the same time, because of the limited capacity, the higher level decision units process more aggregated information than the lower ones. In this paper, a two-level structure related to the supply and transportation layers of a water network is proposed as shown in Fig. 1.

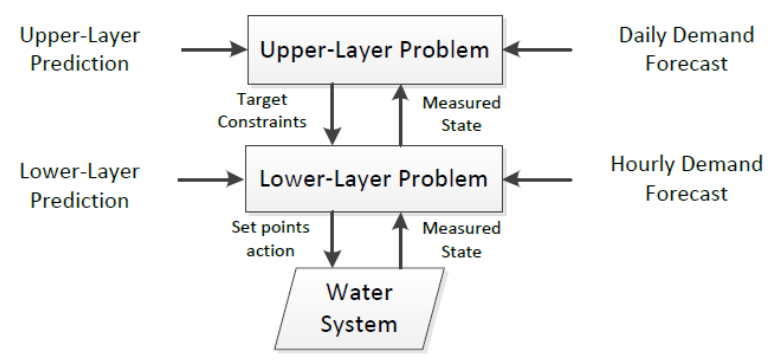

Fig. 1: Temporal hierarchy Multi-layer MPC

The water systems corresponding to these two layers and operated according to different goals and time scales. However, both layers use MPC to compute control strategies which can be characterized by the pair $\left(H_{p}, T_{s}\right)$, where $H_{p}$ is a the time horizon, $T_{s}$ is the sampling time.

\section{Temporal Multi-layer Coordination Techniques}

As shown in Fig. 1, the way to represent interaction between the upper (daily model for the supply layer) and lower (hourly model for the transportation layer) layers relies on two elements:

- Measured disturbance $\left(M_{s}\right)$ : which handles the related aggregated demands at the transportation layer in every horizon time as communication information to the supply layer. 
- Target constraints $\left(T_{d}\right)$ : which expresses management policies at the supply layer to the transportation layer in the form of control constraints.

1) Measured Disturbance: In the topology of the supply layer, the whole transportation layer is simplified as one aggregated demand. Measured state in every optimization process for supply layer is the sum of the related demand in every sampling time of supply layer (here is 24 hours)

$$
M_{s}(k)=\sum_{i=1}^{24} d_{t}(k, i)
$$

where $d_{t}(k, i)$ is demand vector at the transportation layer corresponding to the $k$-th day.

Thus, $M_{s}(k)$ should be considered as the demand for the supply layer

$$
d_{s}(k)=M_{s}(k)
$$

2) Target Constraints: The goal for the temporal coordination algorithm is transferring management policies from the upper (supply) to the lower (transportation) layer. In order to achieve this coordination, the following constraint is added to the the lower layer MPC

$$
\sum_{k=1}^{24} u(i, k) \leq T_{d}(i)
$$

where $u(k)$ are the shared controls between supply and transportation layers.

This constraint is introduced in order to enforce that the amount of water decided to be transferred from the supply to the transportation layer by the upper layer MPC is respected by the lower layer MPC. Without such a constraint the lower layer MPC would decide the amount of water ignoring the upper layer MPC policy.

The coordination working structure is shown at Fig. 2:

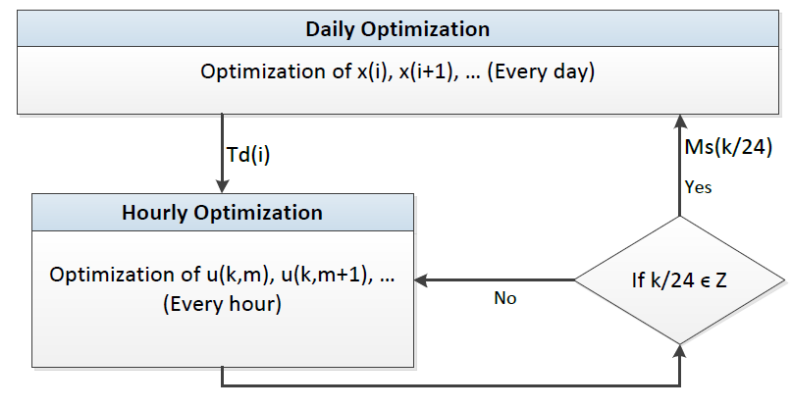

Fig. 2: Upper and Lower layer optimizations of multi-layer MPC

\section{FORMULATION OF THE TEMPORAL MULTI-LAYER MPC SCHEME}

\section{A. State Space Model for Supply Layer}

The state space model of supply layer has two kinds of states and managed variables. First kind of state variables represent reservoirs and the managed variable corresponds to actuator flows:
$x(k+1)=A x(k)+B u(k)+B_{p}[d(k)-\varepsilon(k)], \quad k \in \mathbb{Z}$

where $x(k)$ are state variables representing tank/reservoir volumes, $u(k)$ are the control actions which correspond to actuator flows, $d(k)$ is disturbance corresponds to demands and $\varepsilon(k)$ are the slack variables for violated demands.

At this function, $\varepsilon(k)$ is introduced to control the amount of demand which has not been satisfied.

The second kind of states and managed variable represent river flows in a river reach model with delays. For simplicity and brevity of the explanation, consider river reach model (9) as a transport delay [11]:

$$
q_{\text {out }_{i}}=q_{\text {in }_{i}}\left(k-\tau_{d}\right)
$$

where $\tau_{d}$ represents delay values. For time delays associated with flows within the network, the following auxiliary state equations are introduced:

$$
\begin{gathered}
x_{j, 1}(k+1)=q_{j}(k) \\
x_{j, i+1}(k+1)=x_{j, i}(k), i=1, \cdots, \tau_{d}
\end{gathered}
$$

where $x_{j}, i(k)$ are state variables represent flows, $q_{j}(k)$ mean flows, which are considered as part of managed variables and $\tau_{d}$ is the number of delays.

More details on how this approach can be extended to the case that river reach model (9) is not just considered as a delay can be found in [11].

After integrating (19) and (20) with (17), we have a new augmented state space representation

$$
\widetilde{x}(k+1)=\widetilde{A} \widetilde{x}(k)+\widetilde{B} \widetilde{u}(k)+\widetilde{B}_{p}[d(k)-\varepsilon(k)], \quad k \in \mathbb{Z}
$$

where

$$
\widetilde{x}(k)=\left[\begin{array}{c}
x(k) \\
x_{j, i}(k)
\end{array}\right], \quad \widetilde{u}(k)=\left[\begin{array}{c}
u(k) \\
q_{j}(k)
\end{array}\right]
$$

and $\widetilde{x}(k) \in \mathbb{R}^{\widetilde{n}_{x}}, \widetilde{u}(k) \in \mathbb{R}^{\widetilde{n}_{u}}$.

According to (2) and (3), all the variables are subject to the following inequality constraints:

$$
\begin{aligned}
\widetilde{x}_{\min } & \leq \widetilde{x}(k) \leq \widetilde{x}_{\max } \\
\widetilde{u}_{\min } & \leq \widetilde{u}(k) \leq \widetilde{u}_{\max } \\
\varepsilon_{\min } & \leq \varepsilon(k) \leq \varepsilon_{\max }
\end{aligned}
$$

where $\widetilde{x}_{\min }$ and $\widetilde{x}_{\max }$ are physical limitations of the reservoirs, while $\widetilde{u}_{\min }$ and $\widetilde{u}_{\max }$ are physical limitations of the river flows. The range of $\varepsilon_{\min }$ lies between zero and the related demand.

As described at Section II, balance at every node should be satisfied, where $E, E_{d}, E_{\widetilde{x}}$ are matrices obtained from topology of the water network:

$$
E \widetilde{u}+E_{d} d-E_{d} \varepsilon+E_{\widetilde{x}} \widetilde{x}=0
$$

During the consumption process, water storage in reservoirs should be kept above a given level (named as water safety level) which is used as emergency supply for drought 
periods. Any situation below the emergency level should be penalized using soft constraints:

$$
\begin{gathered}
\widetilde{x} \geq \widetilde{x_{r}}-\varepsilon_{\widetilde{x}} \\
\varepsilon_{\widetilde{x}} \geq 0
\end{gathered}
$$

where $\widetilde{x_{r}}$ is the water safety level and $\varepsilon_{\widetilde{x}}$ is the slack value corresponding to $\widetilde{x_{r}}$.

The state space model of the transportation layer is simpler since the states corresponds to the tank volumes and the manipulated variables are the flows in pumps and valves. This leads to a standard state space representation (10) for the transportation layer. More details can be found in [1].

\section{B. Operational Goals}

1) Operational Goals for Supply Layer: The supply network is operated with a 30-day horizon, using daily time control interval. The main operational goals to be achieved in the supply network are:

- Operational safety $\left(J_{\text {safety }}\right)$ : Maintain appropriate water storage levels in dams for emergency-handling.

- Demand management $\left(J_{\text {demand }}\right)$ : Fully satisfy urban demands while satisfy irrigation demands with slackness.

- Balance management ( $\left.J_{\text {balance }}\right)$ : Keep rivers or reservoirs consumed in a fair and balanced way in order to escape water deficits in the long run.

- Minimizing waste $\left(J_{\text {mwaste }}\right)$ : Minimize unnecessary water release from reservoirs which does not meet any demand and is eventually wasted.

- Environment conservation ( $\left.J_{\text {ecological }}\right)$ : Maintain water levels and ecological flows of rivers, which included in $J_{\text {safety }}$ during the calculation.

Above mentioned goals lead to the following function:

$$
\begin{aligned}
& J=J_{\text {safety }}+J_{\text {demand }}+J_{\text {mwaste }}+J_{\text {balance }} \\
& =\varepsilon_{\widetilde{x}}(k)^{\top} W_{\widetilde{x}} \varepsilon_{\widetilde{x}}(k)+\varepsilon(k)^{\top} W_{f} \varepsilon(k) \\
& +\left(\widetilde{u}_{i \ldots j}(k)-\widetilde{u}_{s}(k)\right)^{\top} W_{\widetilde{w}}\left(\widetilde{u}_{i \ldots j}(k)-\widetilde{u}_{s}(k)\right) \\
& +\left(\left(\begin{array}{lllll}
0 & \ldots & \frac{1}{x i_{\max }^{\prime}} \ldots \frac{-1}{x j_{\max }^{\prime}} \ldots & \ldots
\end{array}\right) \widetilde{x}(k)\right)^{\top} W_{\widetilde{m}} \\
& \times\left(\left(\begin{array}{llll}
0 & \ldots \frac{1}{x i_{\max }^{\prime}} \ldots \frac{-1}{x j_{\max }^{\prime}} \ldots & 0
\end{array}\right) \widetilde{x}(k)\right)
\end{aligned}
$$

where

$$
\begin{aligned}
\varepsilon_{\widetilde{x}}(k) & =\widetilde{x}(k)-\widetilde{x}_{r} \\
\widetilde{u} & =\Theta \Delta \widetilde{u}+\Pi \widetilde{u}(k-1) \\
\Delta \widetilde{u}(k) & =\widetilde{u}(k)-\widetilde{u}(k-1)
\end{aligned}
$$

and $W_{\widetilde{x}}, W_{f}, W_{\widetilde{w}}, W_{\widetilde{x}}, W_{\widetilde{m}}$ are the related weights according to the priority policies established by network managers [9].

2) Operational Goals for Transportation Layer: The transportation network is operated with a 24-hour horizon, at hourly time interval. The main operational goals to be achieved in the transportation network are:

- Cost reduction $\left(J_{\text {cost }}\right)$ : Minimize water cost during water supplying process.
- Operational safety $\left(J_{\text {safety }}\right)$ : Maintain appropriate water storage levels in dams and reservoirs of the network for emergency-handling.

- Control actions smoothness ( $\left.J_{\text {smoothness }}\right)$ : Smooth flow set-point variations for sustainable process operation.

Above mentioned goals lead to the following function:

$$
\begin{aligned}
J & =J_{\text {safety }}+J_{\text {smothness }}+J_{\text {cost }} \\
& =\varepsilon_{\widetilde{x}}(k)^{\top} W_{\widetilde{x}} \varepsilon_{\widetilde{x}}(k)+\Delta \widetilde{u}(k)^{\top} W_{\widetilde{u}} \Delta \widetilde{u}(k) \\
& +W_{a}\left(a_{1}+a_{2}(k)\right) \widetilde{u}(k)
\end{aligned}
$$

where

$$
\begin{aligned}
\varepsilon_{\widetilde{x}}(k) & =\widetilde{x}(k)-\widetilde{x}_{r} \\
\widetilde{u} & =\Theta \Delta \widetilde{u}+\Pi \widetilde{u}(k-1) \\
\Delta \widetilde{u}(k) & =\widetilde{u}(k)-\widetilde{u}(k-1)
\end{aligned}
$$

and $W_{\widetilde{x}}, W_{\widetilde{u}}, W_{a}$ are also related weights.

The vectors $a_{1}$ and $a_{2}$ contain the cost of water treatment and pumping, respectively.

\section{Formulation of the optimization problem}

The objective functions (27) and (28) of the MPC problem can be formulated in the following way:

$$
J=z^{T} \Phi z+\phi^{T} z+c
$$

where

$$
z=\left[\begin{array}{lll}
\Delta \widetilde{u} & \varepsilon_{\widetilde{x}} & \varepsilon
\end{array}\right]^{T}
$$

and $c$ is a constant value.

This allows to determine optimal control actions at each instant $k$ by solving a quadratic optimization problem by means of quadratic programming $(\mathrm{QP})$ algorithm in form as:

$$
\begin{gathered}
\min _{z} z^{\top} \Phi x+\phi^{\top} z \\
A_{1} z \leq b_{1} \\
A_{2} z=b_{2}
\end{gathered}
$$

\section{Formulation of Temporal Coordination Problem}

As explained in Section III, the goal for the temporal coordination algorithm is transferring management policies from the upper (supply) to the lower (transportation) layer. In order to achieve this coordination, the constraint (16) is added to the the lower layer MPC.

Constraints generated after taking into account the following algorithm:

- after the application of $n$ hourly control actions $u_{s}(k)$ corresponding to the $i$-th day, the total remaining water for this day will be: $T_{d}(i)-\sum_{k=1}^{n} u(k)$

- when limiting the control actions in the prediction horizon $L$, there is a part of control actions $u(k)$ that corresponds to hours of the current day $i$ that should be limited by $T_{d}(i)$, while the other part corresponds to hours of the next day $i+1$ that should be limited by $T_{d}(i+1)$.

- the generated constraints are added as additional constraints of the BOP problem associated to the lower layer MPC. 


\section{CASE STUDY: CATALUNYA REGIONAL WATER NETWORK}

\section{A. Description}

The Catalunya Regional Water Network provide the water supplies of the metropolitan area of Barcelona where most of the population of Catalunya region is concentrated. The Catalunya Regional Water Network supplies water mainly from two rivers (Llobregat and Ter).

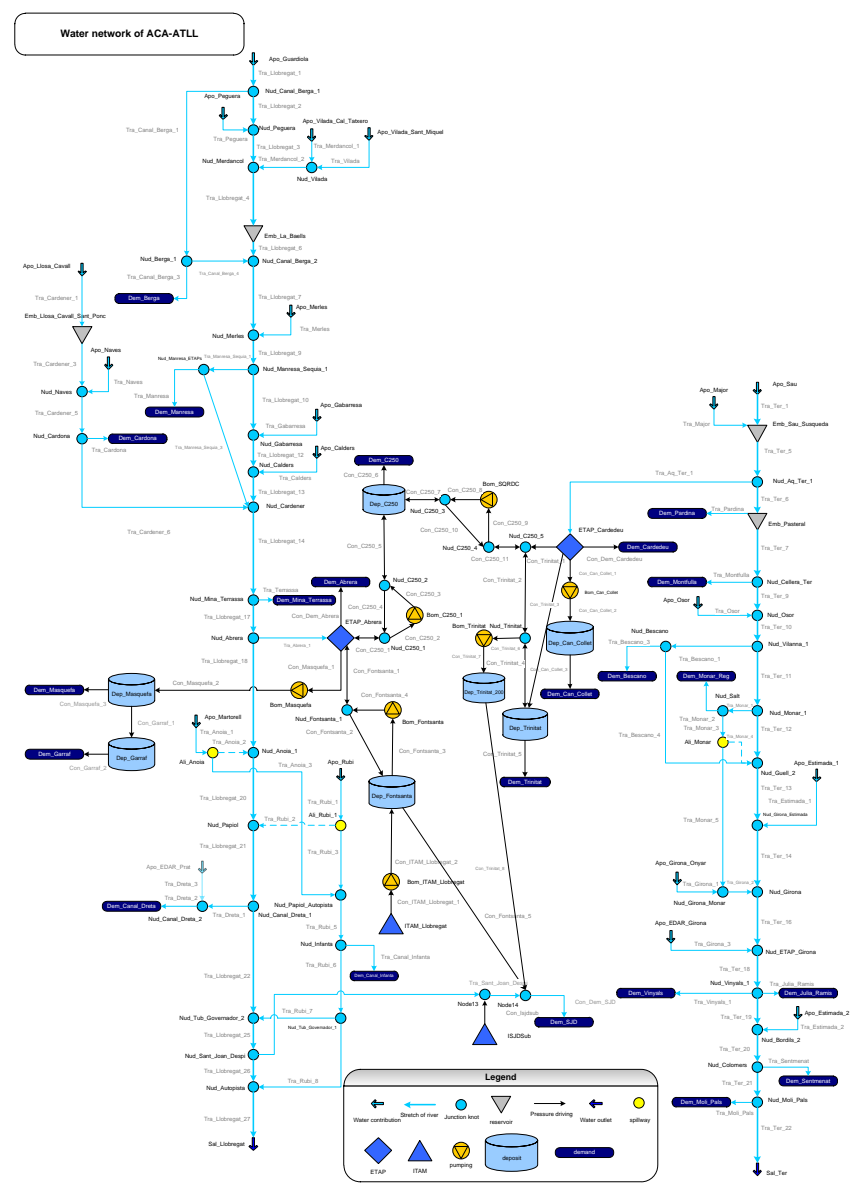

Fig. 3: Aggregate diagram of Catalunya Regional Water Network.

In Fig. 3, an aggregate model of Catalunya Regional Water Network is provided. According to the definition of functional decomposition, the Catalunya Regional Water Network can be separated into three layers. The supply layer, composed by rivers Llobregat, Ter and all the connected elements, lies on the two sides of Fig. 3. The transportation layer, composed by metropolitan areas and also treatment, desalination plants inside them, is in the center of Fig. 3. The distribution layer, which is not described at this network lies inside of each demand at the transportation layer. The hydrological regime of Catalonia, is characterized by the irregularity of its rainfall pattern, which, as is typical of the Mediterranean climate, varies greatly between years. This makes the region especially vulnerable to drought episodes, which are expected to increase due to climate change. This is a big motivation for developing the multi-layer MPC scheme proposed in this paper that provides a better management of the water resources.

\section{B. Control Objectives}

The control objectives of Catalunya Regional Water Network, which are addressed at different layers, are presented in Section IV.

In the Catalunya Regional Water Network, there exists more than one source for some demands. Because of different prices, the demand could ask as much as possible water from the cheaper river, which could lead to overdrawn and unbalanced problem. This paper applies controls that manage water usages at rivers proportionally with the storage capacity of their dams.

\section{RESUlts}

\section{A. Supply Layer}

Table I provides detailed results and also comparison of water usages of the two rivers before and after balancing management achieved with the proposed multi-layer MPC scheme. In the table, $S$ means outside sources flow into rivers, $F D$ means fixed demands which can not choose water source while $V D$ is the demand which can receive water from more than one river. $B D$ is the water volume that has been consumed from each of the reservoirs and $P B$ is proportion of $B D . P R$ is the proportion of storage capacities of the two reservoirs. The similar values for $P B$ and $P R$ is what the balance management wants to reach. And $S A$ is water supply ability in days of the whole water network before meeting deficit problems at the hypothesis of no rain and no water flow in from outside. The comparison proves that, after using balance management, the proportion of water usage from two rivers $(58.93 \%)$ is much more proportional to their storage capacities (53.48\%). And what is more, the Catalunya Regional Water Network can supply water 65 days longer than in the case without balance management, which is a benefit for the sustainable usage of water resource in the long term perspective.

TABLE I: Balancing comparison (all values in e.u.)

\begin{tabular}{llllllll}
\hline Sc. & \multicolumn{7}{c}{ With Balancing Management } \\
\hline Es. & S & FD & VD & BD & PR & PB & SA \\
\hline L. & 3008 & 2981 & 724 & 697 & $58.93 \%$ & $53.48 \%$ & \multirow{2}{*}{242 Days } \\
T. & 3532 & 3518 & 1196 & 1182 & & & \\
\hline Sc. & \multicolumn{7}{c}{ Without Balancing Management } \\
\hline Es. & S & FD & VD & BD & PR & PB & SA \\
\hline L. & 3008 & 2981 & 7.6 & -19.4 & $-1.02 \%$ & $53.48 \%$ & \multirow{2}{*}{177 Days } \\
T. & 3532 & 3518 & 1914 & 1900 & & & \\
\hline
\end{tabular}

\section{B. Transportation Layer}

Fig. 4 shows in the same plot the pump flow compared to its electricity fee. From this figure, it can be noticed that the pump sends more water to the reservoir at the lower price period and less or no water at the higher price period, which is consistency with the economical objective. 


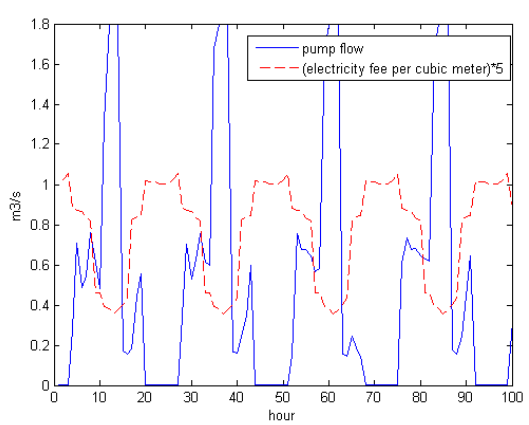

Fig. 4: Pump flow with electricity price

\section{Coordination}

Fig. 5 shows the amount of water consumed by the transportation layer from different rivers for satisfying the same demands before and after coordination, respectively. The two figures prove that average levels of water consumptions from two rivers are much closer after balance management.
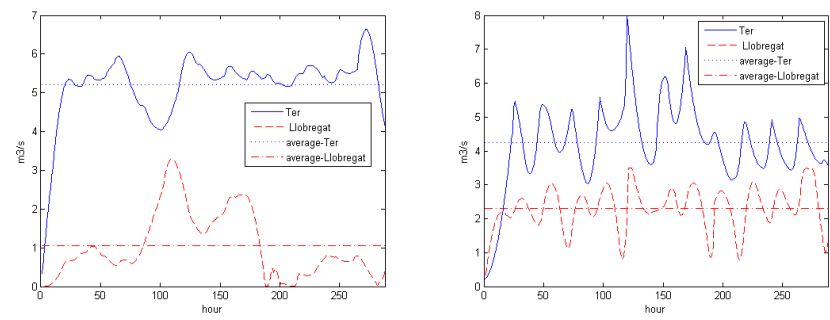

Fig. 5: Flows of two rivers before and after temporal

Table II provides results in detail and compares them in economical cost over four days using three different control techniques: current control, which is the traditional control method without MPC, MPC with temporal multilevel coordination and MPC without coordination.

TABLE II: Closed-loop performance results (all values in e.u.)

\begin{tabular}{ccccccccc}
\hline & Curr. & \multicolumn{4}{c}{ MPC with C. } & \multicolumn{3}{c}{ MPC } \\
Wat. & Ele. & Tot. & Wat. & Ele. & Tot. & Wat. & Ele. & Tot. \\
\hline 240 & 100 & 340 & 213 & 44 & 257 & 141 & 40 & 181 \\
239 & 106 & 345 & 237 & 47 & 284 & 170 & 39 & 209 \\
246 & 94 & 340 & 238 & 48 & 286 & 171 & 41 & 212 \\
264 & 110 & 374 & 253 & 66 & 319 & 168 & 42 & 210 \\
\hline \multicolumn{1}{c}{} & $-5 \%$ & $-50 \%$ & $-18 \%$ & $-34 \%$ & $-61 \%$ & $-42 \%$ \\
\hline
\end{tabular}

Table II shows that, MPC technique with temporal coordination is better than the current control but a little worse than MPC technique without coordination at the point of economical cost, especially regarding water source cost. It is because after introducing coordination techniques, demands at the transportation layer could consume more water from the expensive river in order to keep usage balance. From a long time perspective, sustainable usage and ecological protection of rivers have been achieved at the price of certain limited cost. Besides that, even from an economical perspective, the
MPC with coordination techniques is more desirable than MPC without coordination because after coordination, the Catalunya Regional Water Network can supply water 65 days longer compared to the case without balance management as Table I shows, which saves much economical expenses by solving the deficit problem.

\section{CONCLUSIONS}

In this paper, a multi-layer MPC scheme with multi-level coordination for regional water supply systems is proposed. The need of coordination derives from the fact that different networks in the water supply and transportation systems are operated according to different management goals, with different time horizon. While the management of the supply network is mainly concerned with long term safe-yield and ecological issues, the transportation layer must achieve economic goals in the short term, while meeting demands and operational constraints. The use of the modelling and coordination techniques proposed in this paper allows obtaining short-term strategies which can effectively consider long-term objectives as well.

\section{ACKNOWLEDGMENT}

The authors thank ACA, ATLL and ADASA for providing the case study as well as for sharing their hydrological management expertise. This research has been partially funded by CDTI (MCyT) project HIDROPTIM IDI-20100722, the Spanish research project WATMAN (DPI2009-13744) of the Science and Technology Ministry and the DGR of Generalitat de Catalunya (SAC group Ref. 2009/SGR/1491), the AGAUR by an FI grant and by EFFINET grant FP7-ICT2012-318556 of the European Commission.

\section{REFERENCES}

[1] C. Ocampo-Martinez, V. Puig, G. Cembrano, and J. Quevedo, “Application of predictive control strategies to the management of complex networks in the urban water cycle," IEEE Control Systems Magazine, vol. 33, no. 1, pp. 15-45, 2013.

[2] M. Brdys and B. Ulanicki, Operational Control of Water Systems: Structures, algorithms and applications. UK: Prentice Hall International, 1994.

[3] S. M. de Oca Armeaga, "Hierarchica multilayer and decentralized mpc control of the barcelona water network," Master's thesis.

[4] B. U. M.A. Brdys, Operational Control of Water System. London: Prentice Hall, 1994.

[5] P. V. Overloop, Model Predictive Control on Open Water Systems. Delft, The Netherlands: Delft University Press, 2006.

[6] X. Litrico and V. Fromion, "Simplified modeling of irrigation canals for controller design," Journal of Irrigation and Drainage Engineering, vol. 130, no. 5, pp. 373 - 383, 2004.

[7] J. Schuurmans, "Control of water levels in open-channels," Ph.D. dissertation, Delft University of Technology, 1997.

[8] J. Quevedo, V. Puig, G. Cembrano, and J. Blanch, "Validation and reconstruction of flow meter data in the Barcelona water distribution network," Control Engineering Practice, vol. 11, no. 6, pp. 640-651, June 2010.

[9] J. M. Maciejowski, Predictive Control with Constraints. Great Britain: Prentice Hall, 2002.

[10] M. A. Brdys and P. Tatjewski, Iterative Algorithms for Multilayer Optimizing Control. London: Imperial College Press, 2005.

[11] R. Evans, L. Li, I. Mareels, N. Okello, M. Pham, W. Qiu, and S. K. Saleem, "Real-time optimal control of river basin networks," Preprints of the 18th IFAC World Congress, pp. 11459-11464, 2011. 